\title{
Review and application of Artificial Neural Networks models in reliability analysis of steel structures
}

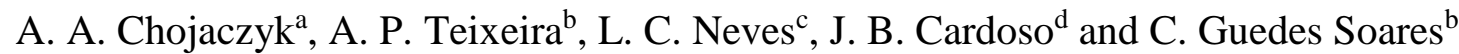

a Research Centre in Structures and Construction (UNIC), Department of Civil Engineering, Universidade Nova de Lisboa, Portugal

b Centre for Marine Technology and Engineering (CENTEC), Instituto Superior Técnico, Universidade de Lisboa, Portugal

c Nottingham Transportation Engineering Centre (NTEC), Dept. of Civil Engineering, University of Nottingham, NG7 2RD Nottingham, United Kingdom

$\mathrm{d}$ Research and Development Unit for Mechanical and Industrial Engineering (UNIDEMI), Department of Mechanical and Industrial Engineering, Universidade Nova de Lisboa, Portugal

\begin{abstract}
This paper presents a survey on the development and use of Artificial Neural Network (ANN) models in structural reliability analysis. The survey identifies the different types of ANNs, the methods of structural reliability assessment that are typically used, the techniques proposed for ANN training set improvement and also some applications of ANN approximations to structural design and optimization problems. ANN models are then used in the reliability analysis of a ship stiffened panel subjected to uniaxial compression loads induced by hull girder vertical bending moment, for which the collapse strength is obtained by means of nonlinear Finite Element Analysis (FEA). The approaches adopted combine the use of adaptive ANN models to approximate directly the limit state function with Monte Carlo simulation (MCS), First Order Reliability Methods (FORM) and MCS with importance sampling (IS), for reliability assessment. A comprehensive comparison of the predictions of the different reliability methods with ANN based LSFs and classical LSF evaluation linked to the FEA is provided.
\end{abstract}

Keywords: artificial neural networks, structural reliability, Monte Carlo simulation, importance sampling, first-order reliability methods, finite element analysis, ultimate strength of stiffened plates.

\section{INTRODUCTION}

The methods for structural safety assessment aim at evaluating the probability of limit state violation by comparing the probabilistic models of acting loads and resistance of a structural component or system. In general, the reliability problem can be formulated by:

$P_{f}=P[g(\mathrm{X}) \leq 0]=\int_{g(\mathrm{X}) \leq 0} f_{\mathrm{X}}(\mathrm{x}) d \mathrm{x}$

where $P_{f}$ is the probability of failure, $g(\mathrm{X})$ is the limit state function (LSF) and $f_{\mathrm{X}}(\mathrm{x})$ is the joint probability density function of the vector $\mathrm{X}=\left\{X_{1}, X_{2}, \ldots, X_{n}\right\}$ of $n$ basic random variables that represent the uncertainty on the material and geometrical properties, structural behaviour and 
loading. Therefore, $g(\mathrm{x})=0$ is $n$-dimensional surface that divides the domain into the safety region $(g(\mathrm{x})>0)$ and failure region $(g(\mathrm{x})<0)($ see Figure 1$)$.

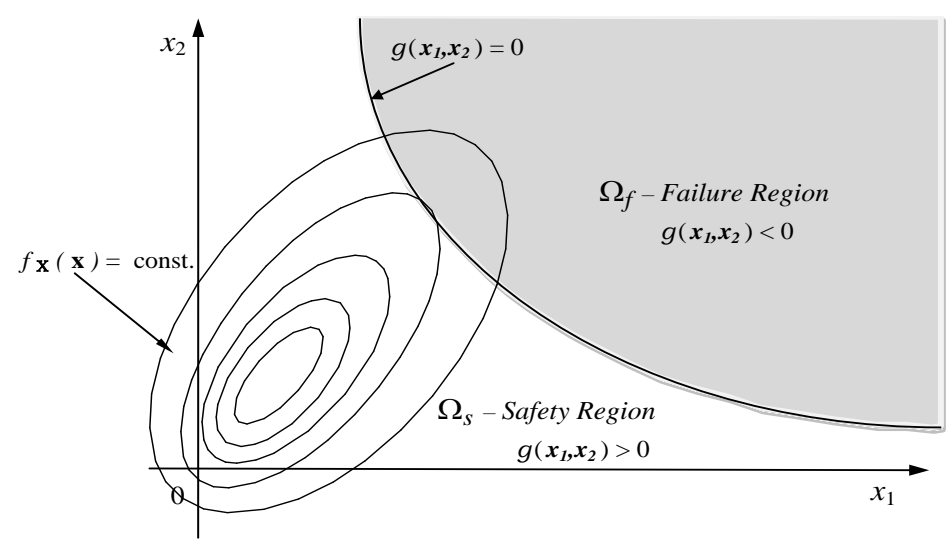

Figure 1 - Basic reliability problem for $n=2$ basic random variables.

Available methods for reliability assessment can be categorized into two main groups: gradientbased and simulation-based methods [1]. The first group consist in an iterative minimization procedure based on the limit state function gradient estimation in order to find the design point, which is a point on the failure surface with the highest probability density, also denoted as the most likely failure point. The distance between the origin and this particular point on the limit state surface is denoted by reliability index $\beta$ [2]:

$u^{*}=\min \{\|u\| \mid g(u)=0\}$ and $\beta=\left\|u^{*}\right\|$

Traditional first-order reliability method (FORM) ([2], [3], [4]) and the second-order reliability method (SORM) ([5];[6];[7];[8]) belong to this class. The simulation techniques have their origin in Monte Carlo simulation (MCS) method, which generates a large sample set of limit state evaluations and approximates the true value of the probability of failure by $P_{f}=\frac{S_{F}}{S}$, where $S_{F}$ is the number of samples lying in the failure region and $S$ the total number of samples. In order to further improve the computational efficiency of MCS, many variance reduction techniques have been proposed [9], including importance sampling ([10], [11]), directional simulation [12] or subset simulation ([13], [14]). Despite these improvements, the MCS method is still timeconsuming and further development is crucial. The above described methods are less suitable for the reliability analysis of complex structures with $g(\mathrm{x})$ defined implicitly, i.e. the evaluation of $g(\mathrm{x})$ requires a time-consuming numerical calculation of the structural response by mean of finite element analysis (FEA). In gradient-based approaches such as FORM, the performance function is approximated by a linear function in a normalized space at the design point and poor accuracy can result from strongly nonlinear performance functions. Moreover, when the LSF has an implicit form, the computational cost of the calculations can be very high. In the simulation methods the problem lays in the enormous number of simulations required for the reliability estimations, since the allowable $P_{f}$ of structures is usually very low.

To overcome these problems, various methods for LSF approximation have been proposed. Among the techniques available to cope with implicit limit state functions, the response surface method (RSM) has proved to be an efficient and widely applicable method in structural reliability analysis. In this method, typically first- or second-order polynomials are chosen to replace the real limit state function ([15];[16];[17];[18]).

Kmiecik and Guedes Soares [19] have used the RSM for probabilistic modelling of the strength of compressed steel plates and, recently, Teixeira and Guedes Soares [20] extended the use of 
this technique to reliability problems involving random fields of corrosion. Gaspar, et al. [21] have combined a response surface approach with a Monte Carlo based simulation method to efficiently solve structural system reliability problems that involve nonlinear finite element analysis.

Artificial neural network (ANN) algorithms introduced as universal function approximations [22] have also been used for structural reliability assessment by several researchers, (e.g. [22], [23], [24], [25], [26]).

Cardoso et al. [27] have shown that ANN is a versatile methodology that can approximate accurately highly non-linear functions over the entire domain with very good precision. Several studies have also been performed showing the accuracy and efficiency of the ANN-based response surface method for reliability assessment in comparison with the conventional response surface methods. Gomes and Awruch [26] indicated that the ANN approach is more efficient, however, the examples considered were relatively simple. More recently, Bucher and Most [24] have applied these approximation methods to several examples of nonlinear structural analysis concluding that the relative accuracy of the various approaches depends on the specific problem under consideration.

The present paper reviews the development and use of ANN models in structural reliability analysis covering the different types of ANNs, the methods of structural reliability assessment that are typically used, the techniques proposed for ANN training set improvement and also some applications of ANN approximations to structural design and optimization problems.

In the second part of this paper, artificial neural network models are applied in the reliability analysis of a ship stiffened panel subjected to uniaxial compression loads induced by hull girder vertical bending moment, for which the collapse strength is obtained by means of nonlinear finite element analysis (FEA). In this application ANN models are used for LSF approximation and combined with Monte Carlo simulation (MCS), first order reliability methods (FORM) and Monte Carlo simulation with importance sampling (MCIS) techniques for reliability assessment. In particular, an adaptive ANN-based MCIS approach using ANN-based FORM for search of the design point is proposed and its efficiency compared with MCIS with LSF evaluation based on direct FEA.

\section{ANN MODELS FOR LIMIT STATE FUNCTION APPROXIMATION}

ANNs are mathematical models based on the neural structure of the brain. ANNs have the capacity of establishing a functional relationship between two data spaces during a learning process and reproduce that connection during a recall process. Various kinds of ANN can be distinguished and many studies on its efficiency and accuracy have been published. The most popular ANN architecture also applied in this study is the multi-layer feed forward network. Herein, only a brief introduction to multi-layer feed forward networks will be presented. More details on different kind of networks and their architecture can be found in literature e.g. [28], [26].

In the ANN, the neuron is a processing element with several inputs and one output [22]. Neuron $m$ receives an input signal vector $\mathrm{X}=\left\{x_{1}, x_{2}, \ldots, x_{n}\right\}$ from $n$ input channels. Next, the weighted sum of $x$ is calculated by multiplying each element $x_{k}$ by a coefficient $w_{m k}$ demonstrating adequate importance of the input channel $k$. The $m$-neuron activation $a_{m}$ is given by:

$a_{m}=\sum_{k=1}^{n} w_{m k} \cdot x_{k}+b_{m}$

where $b_{m}$, called bias, is a constant corrective term which allows having a non-negative activation $a_{m}$, when all elements of the input vector $\mathrm{X}$ are equal 0 . The output signal value $s_{m}$ is 
calculated as a function of the activation, called the transfer function $f\left(a_{m}\right)$. The sigmoid transfer functions are typically used for this purpose and a common choice is the hyperbolic tangent sigmoid transfer function:

$f\left(a_{m}\right)=\frac{2}{1+e^{-2 a_{m}}}-1$

The architecture of a single neuron is shown in Figure 2.

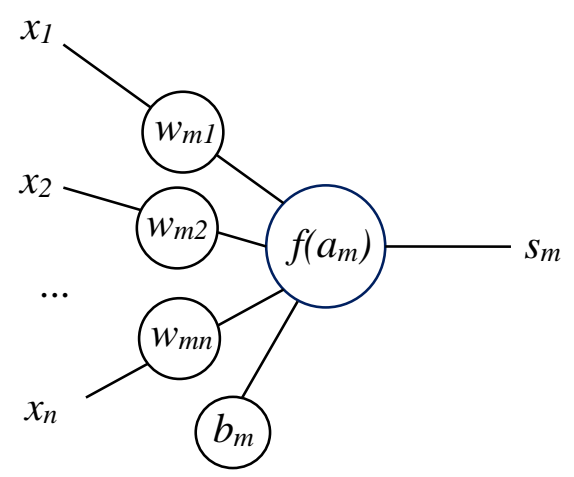

Figure 2 - Artificial neuron

The multi-layer feed forward network consists of various neurons situated on three or more layers - input layer, output layer and one or more hidden layers in between them. The number of neurons on the input layer is equal to the number of input variables while on the output layer it depends on the number of functions to approximate. However, selection of networks optimal architecture is not a simple task and no general rules are applicable for the number of hidden layers and number of the neurons on the hidden layer estimations. In general, higher the complexity of a problem, larger the number of processing elements in hidden layer is needed for a good approximation level and often this is found based on a trial-and-error process. Figure 3 shows the architecture of an example network with 3,4 and 2 neurons respectively on input, hidden and output layer.

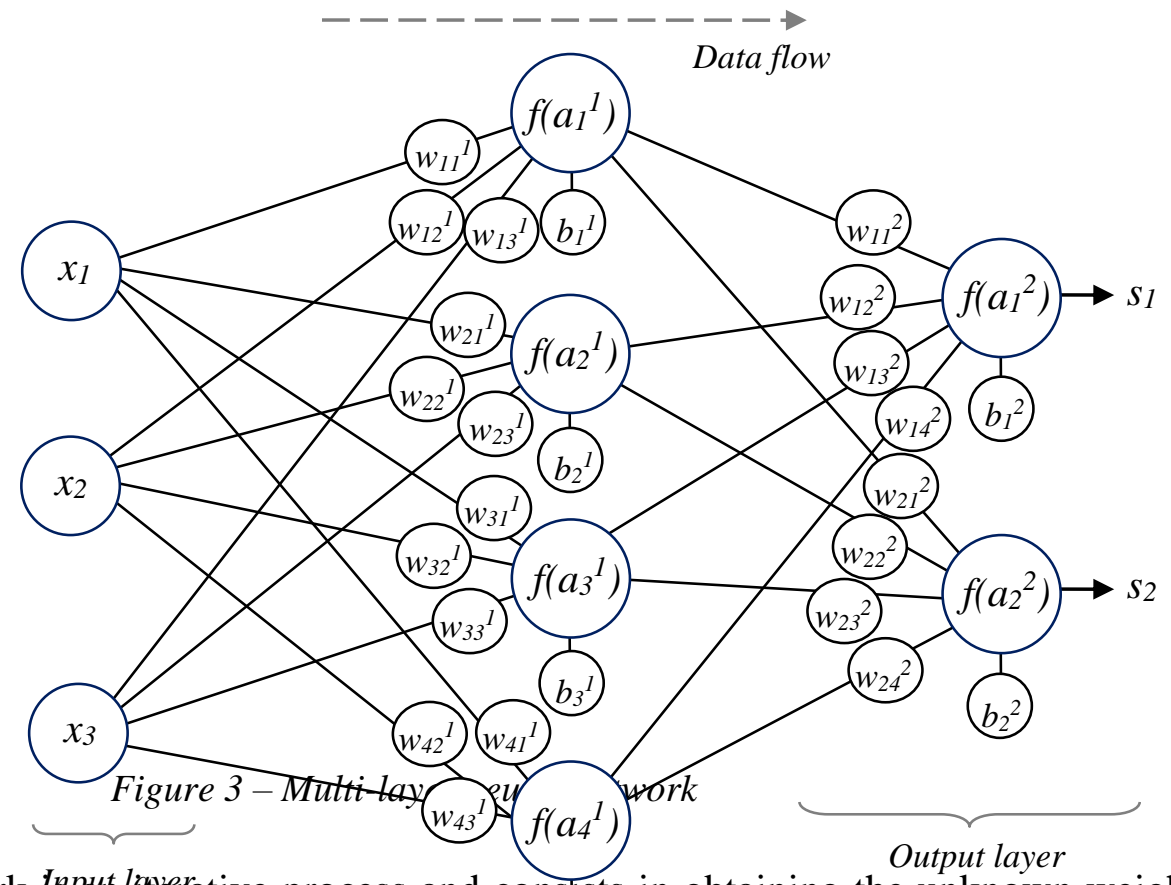

The training of a network Isputh lituerative process and consusts in obtaining the unknown weights $w_{m k}$ and biases $b_{m}$ required for LSF approximation. $b_{4}{ }_{4}$ initial weights and biases are set to 
random values and are subsequently updated by the training algorithm. For this purpose, the training data set with input and target values must be previously prepared. The set is then divided into two sub-sets: 1) the training sub-set which is used for updating the weights and biases and 2) the validation sub-set, used for stopping the training when the network performance fails to improve for previously specified number of iterations or for checking the network approximating capacities. The iterative training algorithm performs an error minimization procedure that is repeated until the network outputs converge to the target values.

The selection of a representative group of samples for training purposes is an important task. To improve training efficiency, each variable should be covered with a sufficient number of samples so that in the recall process the network can approximate the LSF successfully in its entire domain. The size of the sample set grows when the number of variables in the reliability problem rises. Moreover, the samples should be spread over the whole domain for each variable and for this purpose training set improvement techniques can be applied. To start the training process, training data need to be scaled before introducing to network. The scale range depends on the type of the transfer function used. For instance, while using the hyperbolic tangent function the input range is ] $-\infty,+\infty$ [. However, scaling is needed because differences between two very high or low values will result in negligible difference in the function output, which turns the training process difficult. The output values range of $x_{i} \in[-1,1]$, as represented on Figure 4, also need scaling to adapt to the desired function range.

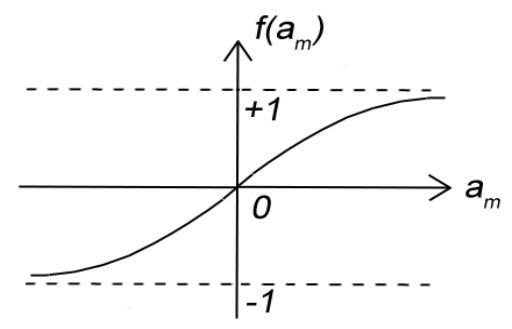

Figure 4 - Hyperbolic tangent function

The default error function used for training feed forward networks is the mean squared error (MSE) - the average squared difference between the network output values $s_{i}$ and the target outputs $t_{i}$, defined as:

$M S E=\frac{1}{N} \sum_{i=1}^{N}\left(t_{i}-s_{i}\right)^{2}$

where $N$ is the number of training samples. One of the problems occurring during ANN training process is called over fitting and it might take place when the number of neurons on hidden layer is excessively large comparing to the number of available training samples. The problem consists in the training error being driven to a very small value while the network still does not learn the LSF correctly. To avoid this problem, $N$ should be greater than the number of unknown weights and bias and can be determined by $(A+C+1) B+C<N$, where $A$ is the number of input values, $B$ is the number of hidden neurons on the single hidden layer and $C$ is the number of functions to approximate [29].

\section{SURVEY OF APPLICATIONS OF ANN IN STRUCTURAL RELIABILITY ANALYSIS}

Despite the fact that the concept of numerical algorithms based on biological neurons was introduced in 1943 by McCulloch et al. [22], the first work that foresees the opportunity of 
applying Artificial Neural Networks (ANN) in structural analysis was published by Hornik et al. (1989) [23]. Since the greatest challenge in reliability analysis is the computation of the limit state function (LSF), research on ANN capability to approximate LSF was performed and several ANN-based structural reliability methods were proposed, compared and modified over the years. An overview of ANN-based reliability methods in a chronological order is presented in Table 1.

\subsection{Types of the ANNs}

ANN can have different architectures that translate into different approximation advantages and disadvantages. For reliability analysis purposes, two most popular networks are used - back propagation multi-layer network and radial basis functions network (RBF). Hurtado and Alvarez [28] tested both and concluded that RBF networks have the following desirable features: high training speed, very small error, accuracy in probability estimation and robustness with respect to changes in model parameters, training sample size and generation procedures. In a subsequent work [1], the same authors analyse, compare and classify not only network types, cost functions, optimization algorithms, sampling methods but also different purposes of use of ANNs. They also recommend procedures for applying ANN in the structural reliability calculations.

Later, Hurtado [30] presented a general application of neural networks in stochastic mechanics and an extensive state of art on the subject, classifying and categorizing network types, purposes and various implementation issues.

\subsection{ANN-based methods of failure probability computation}

Once an adequate surrogate is defined using ANN, it is fundamental to define a methodology to compute the probability of failure compatible with the advantages and limitations of ANN. Both accuracy and computational cost change significantly when using ANN compared to direct evaluation of the LSF, influencing the advantages and disadvantages of each method.

The ANN-based Monte Carlo simulation is a simple and robust method for computing the probability of failure. When combined with ANN, a substantial reduction of the number of finite element calculations can be achieved, as they are only required to build the data set needed for the ANN training process, overcoming a significant disadvantage of traditional MCS. Despite the significant reduction of the computational time, the method is still time consuming and, for this reason, several variance reduction techniques, such as importance sampling or directional simulation, have been widely applied. An extensive description, as well as, numerical examples of the use of ANN-based MCS in structural reliability can be found in ([31], [32], [27], [33], [34]).

Alternatively, gradient-based methods like FORM or SORM can be used in combination with ANN (e.g. [35]). In conventional FORM/SORM, convergence problems might occur while evaluating derivatives using finite differences in case of highly nonlinear limit state function. The use of ANN smoothes the response and reduces the convergence problems, but the obtained results are only an approximation, resulting from the inherit limitations of FORM.

Genetic algorithms (GA) are a search/optimization technique based on the survival of the fittest theory and natural selection proposed by Holland [36]. This method can replace the gradientbased optimization algorithms used in FORM/SORM, but requires a high number of FEA. When using ANN in conjunction with GA, the time of evaluation drops drastically, making GA more effective, as shown by [37], [38], [39]. The ANN-based GA does not need the gradient calculation and overcomes the problem with the calculation of derivatives described before. 
Besides the traditional reliability methods, more innovative methods with use of ANN have been proposed. Nie and Ellingwood [40] used Fekete deterministic point set for the direction identification, while Papadopoulos et al. [14] implemented ANN into subset simulation method for reliability estimations.

\subsection{ANN training set improvement techniques}

In ANN based reliability methods, the quality of LSF approximation and hence, the reliability estimations, are strongly conditioned by the quality of the sample set used for network training. The traditional approach for the training data set preparation involves selection of random samples over the whole domain of each random variable and the iterative process of weight and bias adjustments until the required level of error is obtained. Despite the coarseness of this tactic, it is still one of the most common approaches (e.g. [31], [41], [42]).

In the reliability analysis only a region located near the $g(x)=0$ surface has to be well represented by ANN, as the samples outside of the interest region do not improve considerably the obtained results. In the problems involving complex structures, the region of failure is relatively small when compared with the whole variables domain and, therefore, only a low fraction of samples lays in the region of interest when using random selection. To improve the training process and lower the number of samples needed for the ANN training, iterative training processes were proposed and new approaches for ANN training were developed.

Shaw and Murotsu [43] proposed an active learning algorithm, where the ANN starts training with a limited data set and during this process, evaluates the most critical region. Then, more data is added only in this region and further training is performed, avoiding unnecessary detailed learning at other areas. This process is repeated until a required accuracy is achieved.

Schueremans and Gemert [44] proposed an interesting simulation method based on adaptive ANN model, improved and refined during the reliability analysis. As a result, the number of calls to the LSF remains proportional to the number of random variables. An iterative procedure consisting of adapting ANN and updating the failure probability until required accuracy is reached was proposed. The LSF is only evaluated for samples in the surrounding of the failure region. The ANN is updated as soon as new data become available. The authors use directional sampling or MCIS techniques for reliability calculations. The directional sampling with ANN is more efficient when several directions have a comparable contribution to the failure probability. In other cases, MCIS should be used as it does not require any complex root-finding algorithm.

Cheng et al. [45] proposed an application of the uniform design method (UDM) for the selection of the training data sets in the ANN-based FORM technique. UDM improve quality of selected training data sets, providing the samples scattered over the entire design area as uniformly as possible, turning the set highly representative. The specific feature of UDM is the introduction of the number-theoretic method, which finds a set of points that is scattered over an s-dimensional unit cube and this set is used instead of randomly generated numbers by MCS method. More details on UDM can be found in [46].

Ren and Bai [47] presented two methods for ANN training improvement. In the first method the early stopping point is determined by monitoring the validation error during the training process. The second technique is based on regularization theory and consists in modifying the conventional training performance function by adding the sum of squares of the network weights, so the weights are forced to have smaller values while the training error decreases.

The Latin hypercube sampling was first presented in 1979 by McKay et al. [48] while its implementation into reliability analysis was extensively presented by Olsson et al. [49]. It was 
widely used as an efficiency improvement tool of different importance sampling methods and it also found use in the sample set preparation for ANN training ([33]).

\subsection{Comparison of ANN-based reliability methods}

The main issues in structural reliability assessment are the excessive computational cost as well as the accuracy and applicability of the method to complex structural problems involving implicit limit state function. Many authors described and compared the traditional reliability methods such as MCS, MCIS, FORM, SORM, RSM or empirical method in conjunction with ANN-based reliability methods ([26], [50], [51], [25], [52]).

Deheeger and Lemaire [53] and Tan et al. [54] compared the approximation capacities of ANN and support vector machines, which are based on the structured risk minimization principle and its distinguishing feature lies in ability to define complex decision functions that optimally separate two classes of data samples.

\subsection{Reliability-based structural design and optimization with use of ANN}

Although many efficient methods for reliability calculations were developed and improved, it is still not common to apply them in structural design. The traditional design approach involves the use of empirical equations with partial coefficients calibrated from reliability estimations. This method is very simple and straightforward but, as an approximation technique, can easily lead to over-design of a structure and consequently to an excessive use of materials. Therefore, the ANN based reliability methods can contribute to widespread of reliability analysis into structural design ([55], [56], [57]).

Reliability techniques which use the ANN approximation capacities can be also successfully applied in a large scale structural optimization problems ([58], [59]). 
Table 1 -Review of applications of Artificial Neural Networks in structural reliability analysis review

\begin{tabular}{|c|c|c|}
\hline Authors & Year & Main, innovative ideas \\
\hline $\begin{array}{l}\text { Hornik, } \mathbf{K} \\
\text { Stinchcombe, } M \\
\text { White, } \mathbf{H}\end{array}$ & 1989 & $\begin{array}{l}\text { Prove that standard multilayer feed forward networks are capable of } \\
\text { approximating any measurable function to any desired degree of accuracy. }\end{array}$ \\
\hline $\begin{array}{l}\text { Papadrakakis, M. } \\
\text { Papadopoulos, V. } \\
\text { Lagaros, N. D. }\end{array}$ & 1996 & $\begin{array}{l}\text { Application of ANN in MCS and MCS with IS to the reliability analysis of a } \\
\text { complex structure. Successful implementation of the back propagation } \\
\text { algorithm. }\end{array}$ \\
\hline $\begin{array}{l}\text { Shao, S. } \\
\text { Murotsu, Y. }\end{array}$ & 1997 & $\begin{array}{l}\text { Further development of LSF approximation with use of ANN by introducing the } \\
\text { active learning algorithm to improve learning process, limit data set and achieve } \\
\text { good fitness with the real structure state. }\end{array}$ \\
\hline Hurtado, J. & 2001a & Test and compare the applicability of back propagation multi-layer perceptron \\
\hline Alvarez, D. & $2001 b$ & $\begin{array}{l}\text { and radial basis functions (RBF) networks for reliability assessment of structural } \\
\text { system. }\end{array}$ \\
\hline $\begin{array}{l}\text { Papadrakakis,M. } \\
\text { Lagaros, } \mathrm{N} \text {. }\end{array}$ & 2002 & $\begin{array}{l}\text { Application of ANN to reliability-based structural optimization (RBSO). The } \\
\text { reliability analysis is performed by ANN-based MCS method with IS technique } \\
\text { for the reduction of the sample size. Optimization is performed with evolution } \\
\text { strategies. }\end{array}$ \\
\hline $\begin{array}{l}\text { Gomes, H. } \\
\text { Awruch, A. }\end{array}$ & 2004 & $\begin{array}{l}\text { Test and compare the polynomial-based RSM with ANN-based RSM in terms of } \\
\text { CPU time and the number of LSF evaluations. }\end{array}$ \\
\hline $\begin{array}{l}\text { J. Deng, D. Gu, X. Li } \\
\text { et al. }\end{array}$ & 2004 & $\begin{array}{l}\text { Present and test } 3 \text { methods for structural reliability assessment: ANN-based } \\
\text { MCS, ANN-based FORM and ANN-based SORM. }\end{array}$ \\
\hline J. Nie, & $2004 \mathrm{a}$ & Two types of ANNs are introduced for LSF approximation to facilitate \\
\hline B. Ellingwood & $2004 b$ & $\begin{array}{l}\text { directional simulation-based reliability assessment. The Fekete deterministic } \\
\text { point set is used to identify directions. }\end{array}$ \\
\hline $\begin{array}{l}\text { L. Schueremans, } \\
\text { D. Van Gemert. }\end{array}$ & 2005 & $\begin{array}{l}\text { Proposal of iterative procedure for adapting and refining ANN during the } \\
\text { reliability analysis until required accuracy is reached. The directional sampling } \\
\text { and the MCIS technique used for reliability calculations. }\end{array}$ \\
\hline J. Deng & 2006 & $\begin{array}{l}\text { Present and test RBF ANN-based MCS, RBF ANN-based FORM and RBF } \\
\text { ANN-based SORM, then compare with conventional reliability methods. }\end{array}$ \\
\hline $\begin{array}{l}\text { H. Elhewy, E. } \\
\text { Mesbahi, Y. Pu }\end{array}$ & 2006 & $\begin{array}{l}\text { Implement a standard ANN into MCS for structural reliability evaluations and } \\
\text { provide } 3 \text { numerical examples. }\end{array}$ \\
\hline K. W. Chau & 2007 & $\begin{array}{l}\text { Employ the ANN-based approach to determine performance functions for } \\
\text { reliability and performance-based design. }\end{array}$ \\
\hline J. Cheng & 2007 & $\begin{array}{l}\text { Proposes two ANN-based GA methods: 1) hybrid ANN-based GA method and } \\
\text { 2) hybrid GA method consisting of ANN and MCIS. }\end{array}$ \\
\hline $\begin{array}{l}\text { J. Cheng, } \\
\text { Q. Li, R. Xiao }\end{array}$ & 2008 & Application of UDM to ANN-based FORM for reliability analysis. \\
\hline J. Cheng, Q. Li & 2008 & Application of UDM to ANN-based GA for structural reliability analysis. \\
\hline J. Cheng & 2010 & $\begin{array}{l}\text { Further development of UDM applied to ANN-based GA with penalty function } \\
\text { in a case study of suspension bridge reliability evaluation. }\end{array}$ \\
\hline $\begin{array}{l}\text { X. Tan, W. Bi, X. } \\
\text { Hou, W. Wang }\end{array}$ & 2011 & $\begin{array}{l}\text { Test and compare ANN-based and support vector machine-based RSM with two } \\
\text { different sample selection methods: iterative one and one that do not need } \\
\text { iteration for a sample choice. Probability of failure index is obtained with } \\
\text { FORM. }\end{array}$ \\
\hline $\begin{array}{l}\text { Y. Ren, } \\
\text { G. Bai, }\end{array}$ & 2011 & $\begin{array}{l}\text { Proposal of implementing an early stopping technique and a regularization } \\
\text { theory into ANN-based RSM }\end{array}$ \\
\hline $\begin{array}{l}\text { V.Papadopoulos, } \\
\text { D.Giovanis, } \\
\text { N.Lagaros, } \\
\text { M.Papadrakakis }\end{array}$ & 2012 & $\begin{array}{l}\text { Implementation of ANN into subset simulation method for reliability } \\
\text { estimations. }\end{array}$ \\
\hline
\end{tabular}




\section{ANN-BASED RELIABILITY ANALYSIS OF A STIFFENED PANEL}

An analysis of a deck stiffened panel of a Suezmax oil tanker is performed to assess the capability of the ANN models for reliability assessment. The ship was designed according to Common Structural Rules for Double Hull Oil Tankers [60]. The adopted model was previously considered by Hørte et al. [61] in the development of codes for structural design of ships and later by Gaspar et al. [62], [21] in structural reliability studies. Frequent studies of this type of elements are due to the fact that it is a basic element of a manifold of civil and marine structures.

\subsection{Reliability assessment methodology}

Three different approaches are herein presented and tested, where two of them are ANN-based reliability methodologies that use ANN as the LSF approximator. The first proposed methodology simply combines LSF evaluation by ANN with MCS for failure probability prediction. The Latin hypercube technique is used for training set improvement. The method is tested to predict failure probability of a stiffened steel panel using the ANN to directly approximate the LSF. This approach is very simple and easy to implement in standard software packages, including Matlab [21].

The second approach uses adaptive ANN-based Monte Carlo simulation with importance sampling (MCIS). In this method the ANN is trained in various stages in order to reduce the size of the training set. A first coarse set is established over the whole domain and the ANN is trained. Using this adaptive ANN model an estimate of the design point is obtained by FORM algorithm. Then, additional samples in its neighbourhood are generated and the network is retrained. Afterwards, structural reliability is computed by MCIS, using the iteratively trained ANN

In the above described methodologies, back propagation multi-layer feed-forward networks with one hidden layer, trained with Levenberg-Marquardt ([63],[64]) algorithm are used. The hyperbolic tangent transfer functions are employed in both the hidden and output layers.

The last methodology consists in using FORM and MCIS with standard LSF evaluation via direct coupling with the finite element analysis. This approach is considered very efficient for reliability problems with explicit LSFs and is used as a benchmark for evaluating the accuracy of the ANN-based approaches.

\subsection{Nonlinear finite element structural model}

The stiffened panel of a deck structure of a ship is composed by steel plates, transverse frames and longitudinal stiffeners with T-type cross-section, as presented in Figure 5. Since the panel is symmetric, only one of the stiffeners of the stiffened panel with attached plating is analysed, as shown in Figure 6.

The boundary conditions of the stiffened plate are represented by $\left[u_{x}, u_{y}, u_{z}, r_{x}, r_{y}, r_{z}\right]$, where " 0 " indicates translational or rotational constraint and " 1 " indicates no constraint. For the present analysis, the following boundary conditions are applied:

- $\quad A C$ e $A^{\prime} C^{\prime}$ border (symmetric conditions):

- $\quad A A^{\prime}$ border (symmetric condition):

- $\quad C C^{\prime}$ border (symmetric condition):

- Transverse floors - for plate nodes:

- For stiffener web nodes:

$[1,0,1,0,1,1]$,

$[1,1,1,1,0,0]$,

$[0,1,1,1,0,0]$,

$[1,1,0,1,1,1]$,

$[1,0,1,1,1,1]$. 


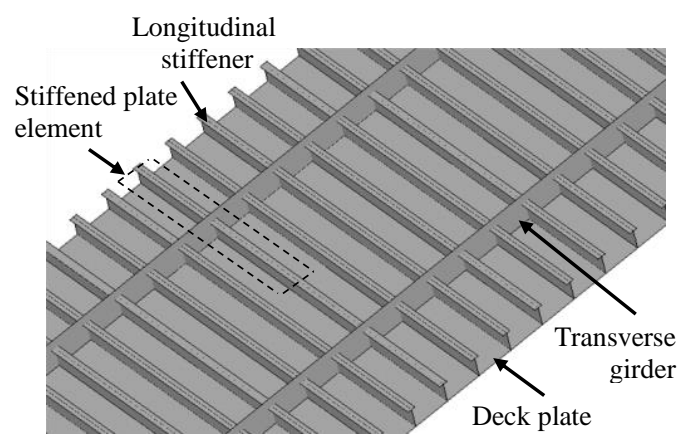

Figure 5 - Deck-stiffened panel

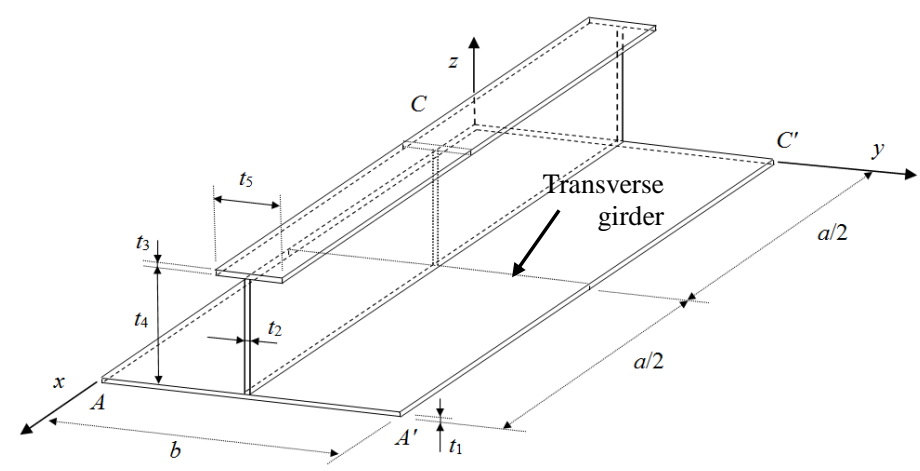

Figure 6-Analysed element of the panel (stiffener with attached plating)

The displacements of nodes on borders $A C$ and $A^{\prime} C^{\prime}$ along the $y$ direction are linked. This procedure ensures uniform displacement even under a point force applied to one of the coupled nodes and also avoids the local distortions of the profile. The design values for the non-corroded condition are given in Table 2, where $a$ is the stiffener span and $b$ is the spacing between stiffeners in the panel. The cross-section geometry is described by the plate $\left(t_{1}\right)$, web $\left(t_{2}\right)$, and flange $\left(t_{3}\right)$ thicknesses, the web height $\left(t_{4}\right)$ and the flange breadth $\left(t_{5}\right)$. When corroded condition is considered, thicknesses $t_{1}, t_{2}$ and $t_{3}$ are reduced by $2 \mathrm{~mm}$ that corresponds to $50 \%$ of the corrosion margin for deck structures as defined in the recent design codes for oil tankers. For the plate material, a high-strength steel $32 \mathrm{AH}$ with yield stress $\sigma_{c}=315 \mathrm{MPa}$, Young's modulus $E=206000 \mathrm{MPa}$ and Poisson's ratio $v=0.3$, is considered.

Table 2- Design dimensions of the stiffened panel $[\mathrm{mm}]$

\begin{tabular}{cc}
\hline Variable & Design Value \\
\hline$a$ & 5450 \\
$b$ & 900 \\
$t_{1}$ & 22.5 \\
$t_{2}$ & 11.5 \\
$t_{3}$ & 16.0 \\
$t_{4}$ & 400 \\
$t_{5}$ & 100 \\
\hline
\end{tabular}

\subsection{Initial deflections}

Welding induced initial deflections with significant contribution to the resistance reduction are considered in the finite element model. The considered pattern is represented in Figure 7, which is composed by the superposition of 4 different deflection shapes equivalent to buckling mode shapes, represented in Figures 7b-7e and given by:

$$
\begin{aligned}
& w_{p}=w_{o p} \cdot \cos \left(\frac{m \pi x}{a}\right) \cdot \cos \left(\frac{\pi y}{b}\right) \\
& w_{w}=w_{o w} \cdot \cos \left(\frac{m \pi x}{a}\right) \cdot \sin \left(\frac{\pi z}{t_{4}}\right) \\
& w_{c}=w_{o c} \cdot \cos \left(\frac{\pi x}{a}\right) \\
& w_{s}=w_{o s} \cdot\left(1-\sin \left(\frac{\pi z}{2 h_{w}}+\frac{\pi}{2}\right)\right) \cdot \cos \left(\frac{\pi x}{a}\right)
\end{aligned}
$$


where $w_{o p}, w_{o w}, w_{o c}$ and $w_{o s}$ are the amplitudes of, respectively, the attached plating deflection, the stiffener web deflection, the stiffener column-type distortion and the stiffener sideways distortion. The design values of those amplitudes are usually assumed to be $w_{p}=b / 200, w_{w}=$ $t_{4} / 200, w_{c}=a / 1000, w_{s}=a / 1000$ according to [60], [65]. The number of buckling halfwaves in longitudinal direction is normally the integer of ratio of longer and shorter side of the plate $(m=a / b)$, and here is considered as $m=7$. The finite element model with the combined shape of initial distortions implemented is shown on Figure 7 a (with a scale factor $\times 50$ ).

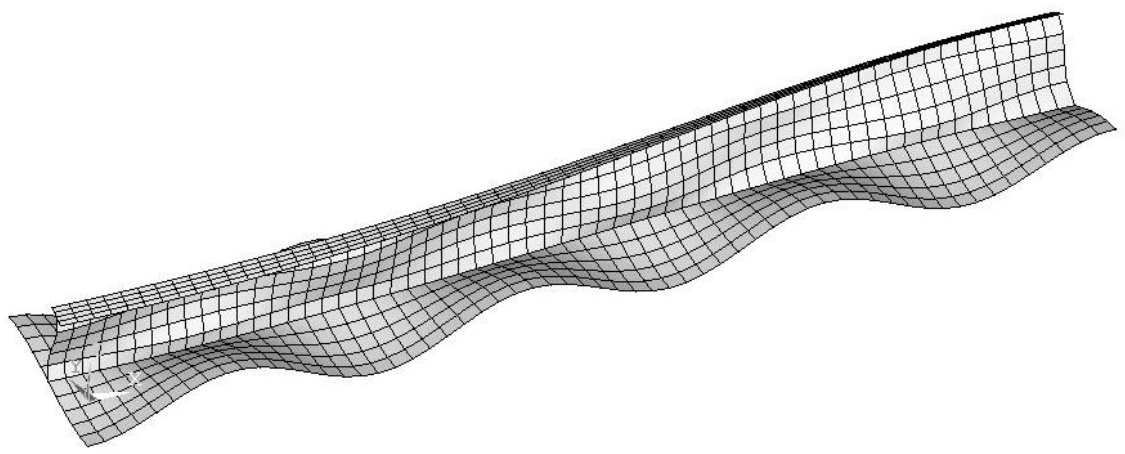

a) Combined geometrical deflection

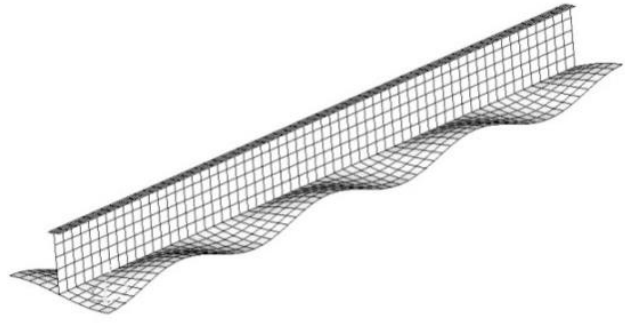

b) Plate geometrical deflection

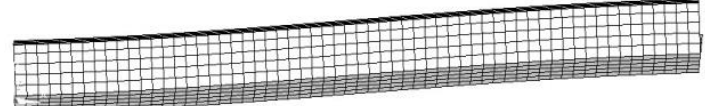

d) Beam-column type geometrical deflection

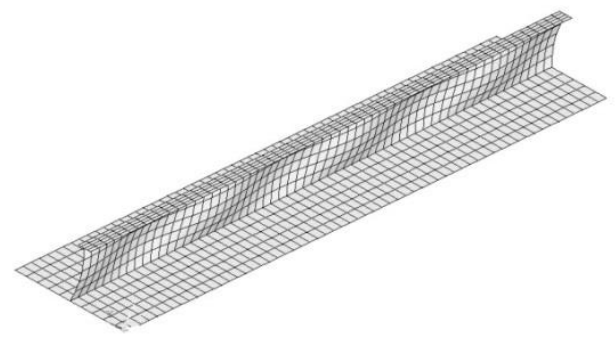

c) Stiffener web geometrical deflection

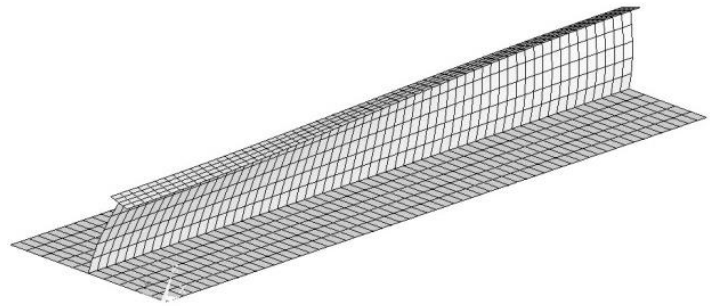

e) Sideways geometrical deflection

Figure 7 - Initial geometrical distortions of the stiffened plate

\subsection{Probabilistic models of basic random variables}

13 random variables associated with strength (Table 3) and two related to load actions (Table 4) are considered in the reliability analysis.

The probabilistic models adopted for the geometrical and material properties of the structural element are well described in the literature [66] and were used in previous studies [21]. The design amplitudes of the welding induced imperfections correspond to the $95^{\text {th }}$ percentile and the coefficient of variation (COV) is assumed as equal 0.50 . 
Table 3-Stochastic models of the strength basic random variables (* - values corresponding to the corroded state)

\begin{tabular}{cccccc}
\hline Variable & Units & Probabilistic Distribution & Mean Value, $\mu$ & COV & Standard Deviation, $\sigma$ \\
\hline$t_{1}$ & $\mathrm{~mm}$ & Normal & $22.5(20.5)^{*}$ & 0.02 & $0.45(0.41)^{*}$ \\
$t_{2}$ & $\mathrm{~mm}$ & Normal & $11.5(9.5)^{*}$ & 0.02 & $0,23(0.19)^{*}$ \\
$t_{3}$ & $\mathrm{~mm}$ & Normal & $16.0(14.0)^{*}$ & 0.02 & $0.32(0.28)^{*}$ \\
$t_{4}$ & $\mathrm{~mm}$ & Normal & 400 & 0.01 & 4.0 \\
$t_{5}$ & $\mathrm{~mm}$ & Normal & 100 & 0.03 & 3.0 \\
$E_{p}$ & $\mathrm{MPa}$ & Lognormal & 206000 & 0.10 & 12.36 \\
$E_{s}$ & $\mathrm{MPa}$ & Lognormal & 206000 & 0.10 & 12.36 \\
$\sigma_{c p}$ & $\mathrm{MPa}$ & Lognormal & 348 & 0.08 & 20.88 \\
$\sigma_{c s}$ & $\mathrm{MPa}$ & Lognormal & 348 & 0.08 & 20.88 \\
$w_{o p}$ & $\mathrm{~mm}$ & Lognormal & 2.30 & 0.50 & 1.15 \\
$w_{o w}$ & $\mathrm{~mm}$ & Lognormal & 1.00 & 0.50 & 0.50 \\
$w_{o c}$ & $\mathrm{~mm}$ & Lognormal & 2.80 & 0.50 & 1.40 \\
$w_{o s}$ & $\mathrm{~mm}$ & Lognormal & 2.80 & 0.50 & 1.40 \\
\hline
\end{tabular}

The probabilistic models adopted for the hull girder vertical bending moments are defined based on work of Horte et al. [61] and as specified in IACS [60]. The still water bending moment $\left(M_{s w}\right)$ is described by a normal distribution with mean equal to $\mu=0.7 \cdot M_{s w, \max }$ and standard deviation $\sigma=0.2 \cdot M_{s w, \max }$, where $M_{s w, \max }=2119.6 \mathrm{MNm}$ is the maximum still water bending moment specified in the ship loading manual.

The long-term probability distribution of the vertical wave-induced bending moment is represented by a Weibull distribution with shape parameter equal to 1 and scale parameter satisfying: $P\left[M_{w v}>M_{w v, \max }\right]=10^{-8}$, where $M_{w v, \max }$ is the maximum vertical wave-induced bending moment considered for design purposes [60]. The Weibull distribution represents the vertical wave-induced bending moment at a random point in time. The peak values over the reference time period $\left(T_{r}\right)$ of 1 year are then described by the Gumbel distribution. Table 4 shows the stochastic models for load basic random variables, more details can be found in [61] and [21].

Table 4 - Stochastic models of the load basic random variables.

\begin{tabular}{cccccc}
\hline \multirow{2}{*}{ Variable } & Units & Probabilistic Distribution & Mean Value, $\mu$ & COV & Standard Deviation, $\sigma$ \\
\hline$M_{s w}$ & MNm & Normal & 1483.700 & 0.29 & 430.300 \\
$M_{w v}$ & MNm & Gumbel & 4603.153 & 0.09 & 401.181 \\
\hline
\end{tabular}

The compressive loading on the stiffened plate of the ship deck is calculated from the total vertical bending moment and the mid-ship section modulus at deck equal to $Z=41.1 \mathrm{~m}^{3}$, for the intact scantlings and $Z=37.3 \mathrm{~m}^{3}$, for the corroded scantlings. The longitudinal compressive load $\left(\sigma_{a}\right)$ applied to the ship stiffened plate is calculated as follows:

$\sigma_{a}=\frac{M_{s w}+M_{w v}}{Z}$

The limit state function $g(\mathrm{X})$ used in the reliability analysis corresponds to the buckling failure of the ship stiffened plate under uniaxial compressive loading, given by:

$g(\mathrm{X})=\sigma_{c o l}-\sigma_{a}$ 
where $\sigma_{c o l}$ is the ultimate compressive strength of the stiffened plate. In this study the ANN predicts directly the LSF, $g(\mathrm{X})$.

\section{RESULTS OF THE RELIABILITY ANALYSIS}

A sample set created with Latin hypercube technique is used for the ANN-based reliability methodologies. The finite element analysis program ANSYS is used to perform the non-linear analysis and to compute the collapse strength of the stiffened plate.

Figure 8 shows the stress-strain curve obtained assuming the mean value of the basic random variables. The ultimate compressive strength, defined as the maximum value of the stress-strain curve, is $\sigma_{c o l}=283.36 \mathrm{MPa}$. The non-linear finite element analysis for each sample takes 3.71 second. Parallel computing technique with 4 dual-core processors has been used, reducing significantly the computational time, when compared with the sequential version.

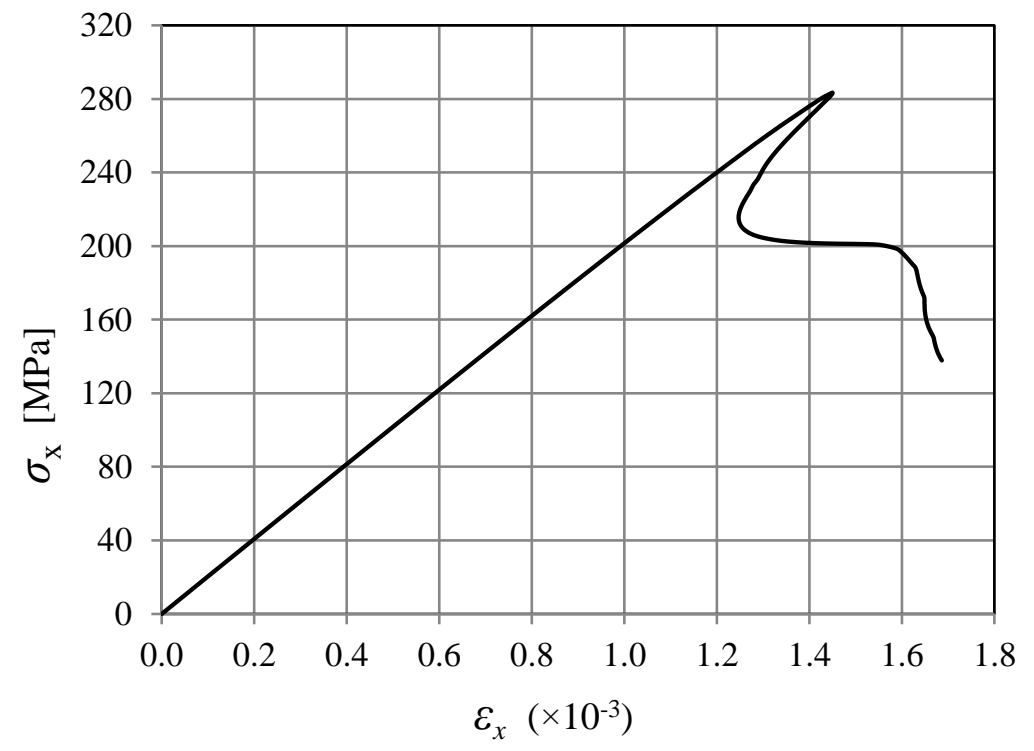

Figure 8-Average stress-strain curve for the mean value of the strength basic random variables for the corroded condition.

\subsection{ANN-based reliability analysis by Monte Carlo simulation}

The results of the first method that consists of using ANN for LSF approximation and MCS for reliability assessment, can be found in Table 5. Training sets of different dimension, ranging from 2000 to 20000, have been considered to check their influence on the ANN approximation capacities and the reliability analysis errors. The results from Table 5 show a trade-off between computational time and accuracy, as presented in Figure 9. The best results, with error considered as equal 0 , were obtained with the largest sample set. However, the preparation of the training set can be highly time consuming, as it is proportional to the number of samples required. Therefore, for computational cost reduction purposes an intermediate value of 10000 elements can be chosen, which ensures the error of the ANN around 1\%. A very efficient Levenberg-Marquard training algorithm has been used and the time consumed for the ANN training is considered as irrelevant to this study. 


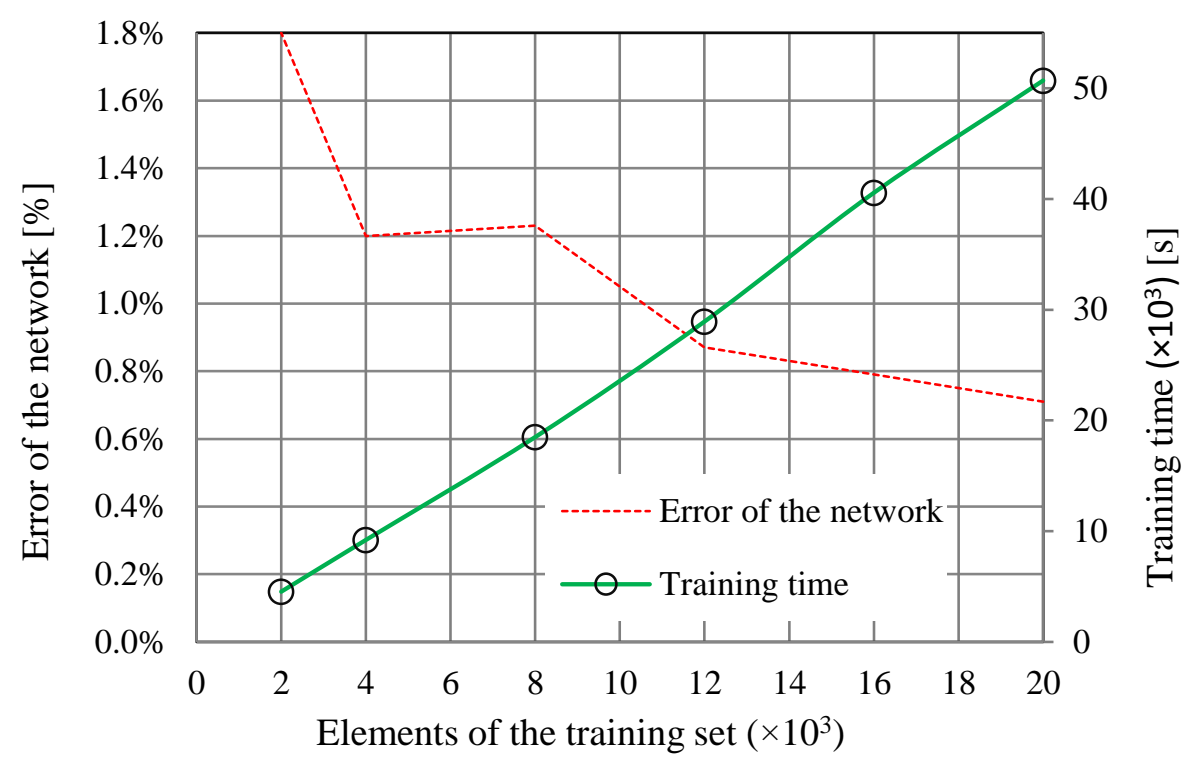

Figure 9-Time spent on the training set preparation and ANN accuracy as function of the size of the training set.

After the ANN has been successfully trained, the ANN-based MCS was performed for reliability index prediction. The number of LSF simulations needed to evaluate the probability of failure with a required precision depends on its order of magnitude. According to Shooman [67], the accuracy of MCS can be measured by coefficient of variation $(\mathrm{COV})$ of the probability of failure $\left(P_{f}\right)$ given by:

$\operatorname{COV}\left(P_{f}\right)=\frac{1}{P_{f}} \sqrt{\frac{\left(1-P_{f}\right) P_{f}}{n_{M C S}}}$

where $n_{M C S}$ is the number of MCS samples. In the present case, $n_{M C S}=1 \times 10^{8}$ was adopted, which corresponds to $\operatorname{COV}\left(P_{f}\right) \approx 2.5 \%$.

Figure 10 shows the variation of the calculated reliability index $(\beta)$ and confidence intervals $(\mathrm{CI})$ with the number of elements in the training set. The estimated failure probability computed by the first methodology converges with the increase of the elements in the training set to $P_{f}=1.97 \times 10^{-5}$, which corresponds to the reliability index $\beta=-\phi^{-1}\left(P_{f}\right)=4.11$.

The CI curves presented in Figure 10 were obtained using a procedure inspired by the bootstrap technique. The basic bootstrap technique uses a sample set as a pseudo-population in order to resample and obtain statistic measures [68], [69]. In this case, the bootstrap technique could not be applied for the CI estimations as it is incompatible with the Latin hypercube methodology implemented in the ANN-based approach, crucial for the ANN training efficiency.

In order to overcome this difficulty, an ANN trained with a very large sample set was assumed to represent the true LSF. Then, different sample sets obtained with Latin hypercube were generated and the corresponding LSF values retrieved. Estimations of mean and standard deviation are used for the CI (95\%) of the reliability index calculations. As show in Figure 10, the CI becomes narrower as the number of elements in the set increases due to the effect of reducing standard error of the probability of failure estimations by MCS. The best prediction of the reliability index is obtained with use of ANN trained with the largest set of 20000 samples, corresponding to a CI of [4.08, 4.17]. 
Table 5- Times and errors of ANN training and results of the ANN-based MCS reliability analysis

\begin{tabular}{cccc|cccc}
\hline \multicolumn{4}{c|}{ Artificial Neural Network } & \multicolumn{4}{c}{ Reliability Analysis } \\
\hline $\begin{array}{c}\text { Training } \\
\text { set }\end{array}$ & $\begin{array}{c}\text { Max. relative } \\
\text { error }\end{array}$ & $\begin{array}{c}\text { Mean square } \\
\text { error }\end{array}$ & $\begin{array}{c}\text { Time of } \\
\text { FEA }\end{array}$ & $P_{f}$ & $\beta=-\Phi^{-1}\left(P_{f}\right)$ & $\begin{array}{c}\text { Relative } \\
\text { error } \\
{[\%]}\end{array}$ & $\begin{array}{c}\text { Time of the } \\
\text { simulation }\end{array}$ \\
\hline 20000 & $0.71 \%$ & $2.26 \times 10^{-4}$ & $50694 \mathrm{~s}$ & $1.970 \times 10^{-5}$ & 4.111 & $\sim 0$ & $\sim 21 \mathrm{~h}$ \\
16000 & $0.79 \%$ & $9.67 \times 10^{-5}$ & $40558 \mathrm{~s}$ & $1.980 \times 10^{-5}$ & 4.110 & 0.508 & {$[4$ processors $]$} \\
12000 & $0.87 \%$ & $1.20 \times 10^{-4}$ & $28934 \mathrm{~s}$ & $1.893 \times 10^{-5}$ & 4.120 & 3.909 & $\sim 17 \mathrm{~h}$ \\
8000 & $1.23 \%$ & $1.82 \times 10^{-4}$ & $18499 \mathrm{~s}$ & $2.071 \times 10^{-5}$ & 4.099 & 5.127 & {$[6$ processors $]$} \\
4000 & $1.20 \%$ & $2.28 \times 10^{-4}$ & $9210 \mathrm{~s}$ & $1.262 \times 10^{-5}$ & 4.213 & 35.939 & $\sim 12 \mathrm{~h}$ \\
2000 & $1.80 \%$ & $3.01 \times 10^{-4}$ & $4542 \mathrm{~s}$ & $1.782 \times 10^{-5}$ & 4.134 & 9.543 & {$[10$ processors $]$} \\
\hline
\end{tabular}

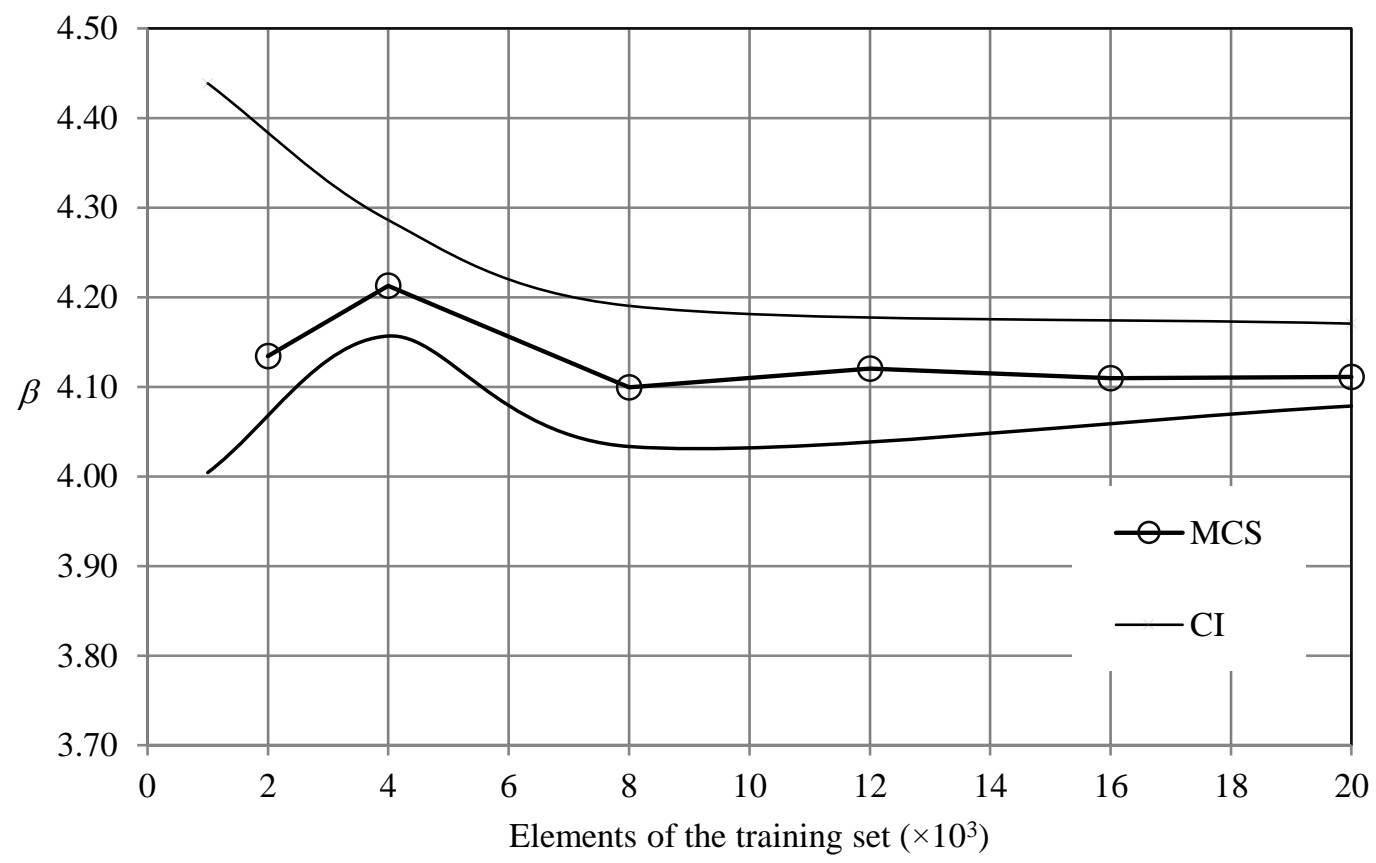

Figure 10 - Estimated probability of failure as a function of the number of elements in the training data set.

\subsection{ANN-based reliability analysis by MCIS}

The efficiency of the direct MCS method depends on the magnitude of the failure probability, i.e. smaller failure probabilities, which usually appear in structural reliability, require larger numbers of samples. This drawback of MCS method can be overcome by generating samples on the base of a different probability density function so that a large number of outcomes fall in the failure domain. This technique is called importance sampling and the related probability density function $h_{\mathrm{X}}(\mathrm{x})$, is denoted as importance sampling density. The failure probability given by Eq. 1 can be rewritten, without losing the generality, as:

$P_{f}=\int_{g(\mathrm{X}) \leq 0}\left(\frac{f_{\mathrm{X}}(\mathrm{x})}{h_{\mathrm{X}}(\mathrm{x})}\right) h_{\mathrm{X}}(\mathrm{x}) d \mathrm{x}$

The efficiency of this technique depends on the selection of $h_{\mathrm{X}}(\mathrm{x})$. Importance sampling is generally recognized as the most efficient variance reduction technique. A successful choice of $h_{\mathrm{x}}(x)$ yields reliable results and reduces significantly the number of simulations, while an inappropriate choice produces inaccurate results. 
Many different suggestions of sampling distributions can be found in the literature. The best of these choices are supported on information from FORM or SORM analyses about where the most essential contributions to the failure probability are located.

If sampling is made around the most probable point of failure $\mathrm{u}^{*}$, evaluated previously from a FORM/SORM analysis, the density function $h_{\mathrm{U}}(\mathrm{u})$ in the standard normal space is the joint probability density function of the basic random variables $\varphi_{\mathrm{U}}(\mathrm{u})$ centred in $\mathrm{u}^{*}$, as illustrated on Figure 11 and given by:

$$
h_{\mathrm{U}}(\mathrm{u})=\frac{1}{(2 \pi)^{n / 2}} \exp \left[-\frac{1}{2}\left\|\mathrm{u}-\mathrm{u}^{*}\right\|\right]
$$

The relative standard error of IS technique is calculated by:

$\delta=\frac{1}{\hat{p}_{f}} \sqrt{\frac{1}{n_{M C I S}\left(n_{M C I S}-1\right)} \sum_{i=1}^{n_{M C I S}}\left(\mathrm{I}(g(\mathrm{x})) \frac{f(\mathrm{x})}{h(\mathrm{x})}-\hat{p}_{f}\right)^{2}}$

where $n_{M C I S}$ is the number of IS simulations and $\hat{p}_{f}$ is the failure probability estimated with use of MCIS. More details on procedure of MCIS can be found in e.g. [70].

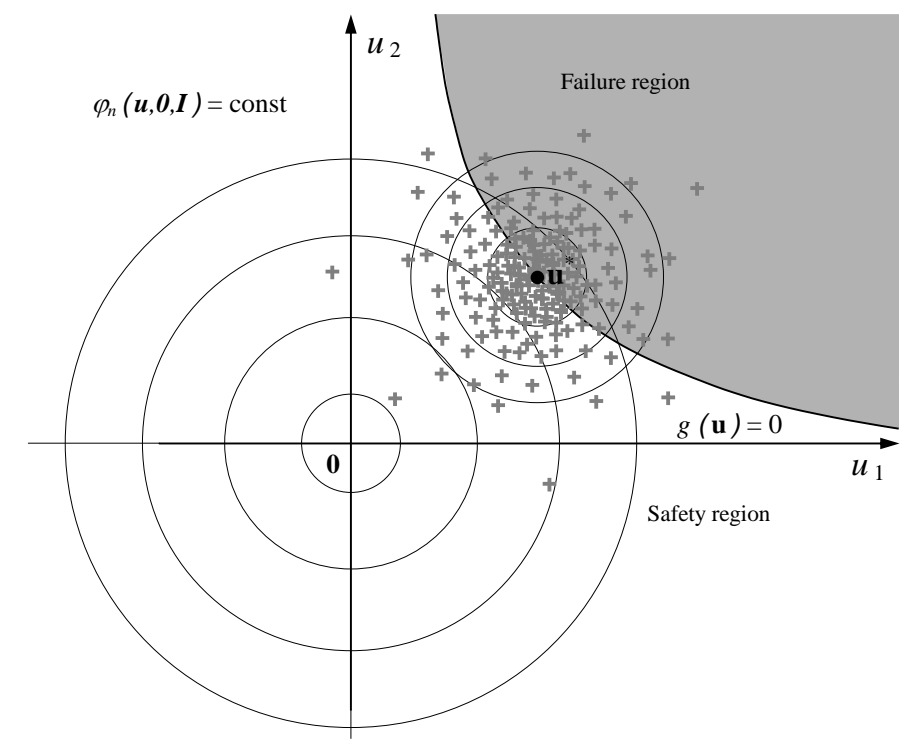

Figure 11- Monte Carlo simulation method with importance sampling

In this work, the importance sampling method is applied based on an adaptively trained ANN. In a first step the network is trained over the whole domain with a reduced number of samples generated using the Latin hypercube method. Three different ANNs with 500, 250 and 100 training samples were tested. The trained ANN is then used to find the design point $\mathrm{u}^{*}$ and the related reliability index ( $\beta$ FORM) by FORM algorithm. In the second step more samples in the surrounding of design point are created and added to the previous training set. In this process 500, 250 and 100 samples are generated over a hypercube with a reduced size domain of width equal to the standard deviation and centred at the FORM-design point. The re-trained ANN is used in the ANN-based FORM algorithm to obtain a new design point. This process is repeated until the new design point is found in the hypercube used in the previous step iteration. This stopping criterion ensures that the new samples are generated in the region of real interest and the network is sufficiently well trained. Afterwards, structural reliability is computed using the successfully trained ANN with MCIS around the most recent design point.

In the present case, a satisfactory level of ANN approximation is obtained after performing only one iteration of the second step, in all cases. Table 6 presents the results of this approach in terms 
of error of the ANN limit state function approximations and reliability indices obtained for each step.

The estimated failure probability converges to the value $P_{f \text { MCIS }}=2.07 \times 10^{-5}$, which corresponds to $\beta_{\text {MCIS }}=4.100$ calculated based on a training set of $500+500$ samples. In this case, the computational time of the analysis has been reduced to $3143 \mathrm{~s}$. The average standard error of IS remains lower than $12 \%$ when the number of ANN evaluations in MCIS is higher than 1000.

Table 6- Results of the ANN-based MCIS

\begin{tabular}{|c|c|c|c|c|c|c|c|}
\hline \multirow{3}{*}{$\begin{array}{l}\text { Training set } \\
\text { (Step1+Step2) }\end{array}$} & \multicolumn{4}{|c|}{ ANN-training } & \multicolumn{2}{|c|}{$\begin{array}{c}\text { Reliability Analysis } \\
\text { (FORM+MCIS) }\end{array}$} & \multirow{3}{*}{$\begin{array}{c}\text { Total } \\
\text { estimation time } \\
{[\mathrm{s}]}\end{array}$} \\
\hline & \multicolumn{2}{|c|}{ ANN error [\%] } & \multicolumn{2}{|c|}{$\beta_{\text {FORM }}$} & \multirow{2}{*}{$\begin{array}{l}P_{f} \mathrm{MCIS} \\
{\left[\times 10^{-5}\right]}\end{array}$} & \multirow{2}{*}{$\beta_{\text {MCIS }}$} & \\
\hline & Step 1 & Step 2 & Step 1 & Step 2 & & & \\
\hline $500+500$ & 2.3 & 0.9 & 2.070 & 4.329 & 2.07 & 4.100 & 3143 \\
\hline $250+250$ & 3.2 & 1.0 & 2.008 & 4.374 & 2.01 & 4.107 & 1717 \\
\hline $100+100$ & 7.4 & 2.3 & 2.381 & 4.365 & 1.25 & 4.215 & 693 \\
\hline
\end{tabular}

\subsection{Monte Carlo Simulation with importance sampling using FEA-based LSF}

The last reliability assessment approach consists in using FORM and MCIS with standard LSF evaluation via direct coupling with the finite element analysis. In this case the calls of limit state function are transferred to the FEA and the results are passed back to the reliability processor.

Table 7 presents the results of structural reliability analysis obtained by FORM and Monte Carlo simulation with importance sampling (MCIS). The reliability index $\beta_{\text {FORM }}$ is obtained with five iterations of the FORM algorithm corresponding to $n_{\mathrm{FORM}}=85$ calculation of the limit state function and hence of the ultimate strength of the stiffened plate by FEA.

Several IS sample sizes ( $n_{\text {MCIS }}$ ) in the region around the design point obtained by FORM, have been considered and, therefore, the total number of calculations of the limit state function is $n_{\text {FORM }}+n_{\text {MCIS. }}$ Table 7 shows that $\beta_{\text {FORM }}=4.270$ and that more accurate estimates can be obtained as the number of IS simulations ( $\left.n_{\mathrm{MCIS}}\right)$ increase.

Figure 12 clearly shows that the reliability index decreases and stabilizes with the increase of $n_{\text {MCIS. }}$ Furthermore, the figure also shows that the $95 \%$ confidence intervals (CI) of the reliability index also tend to become narrower as the number of simulations increase due to the effect of reducing standard error of the probability of failure estimated by importance sampling.

The best prediction of the reliability index is $\beta_{\text {MCIS }}=4.098$, obtained with $1085\left(n_{\text {FORM }}+n_{\text {MCIS }}\right)$ evaluations of the limit state function. In this case, the confidence interval is [4.06, 4.14], which corresponds to a change of only $-4 \%$ on the reliability index $\beta_{\text {FORM }}=4.270$ obtained with only 85 calculations of the function limit state.

Table 7- Results of the Monte Carlo simulation with importance sampling (MCIS) using FEA-based LSF

\begin{tabular}{l|ccccc}
\hline \multicolumn{5}{c}{ FORM $\left(n_{\mathrm{FORM}}=85\right)$} & \multicolumn{2}{c}{$\beta_{\text {FORM }}=4.270$} & \multicolumn{2}{c}{$P_{f}=9.78 \times 10^{-6}$} \\
\hline \multicolumn{2}{c}{ Monte Carlo simulation with importance sampling $(\mathrm{MCIS})$} \\
\hline$n_{\text {MCIS }}$ & 100 & 200 & 400 & 600 & 1000 \\
\hline$\beta_{\text {MCIS }}$ & 4.232 & 4.139 & 4.097 & 4.120 & 4.098 \\
$P_{f}$ MCIS & $1.16 \times 10^{-5}$ & $1.75 \times 10^{-5}$ & $2.09 \times 10^{-5}$ & $1.90 \times 10^{-5}$ & $2.09 \times 10^{-5}$ \\
$\mathrm{COV} p_{f}$ & 0.247 & 0.153 & 0.120 & 0.091 & 0.080 \\
\hline
\end{tabular}




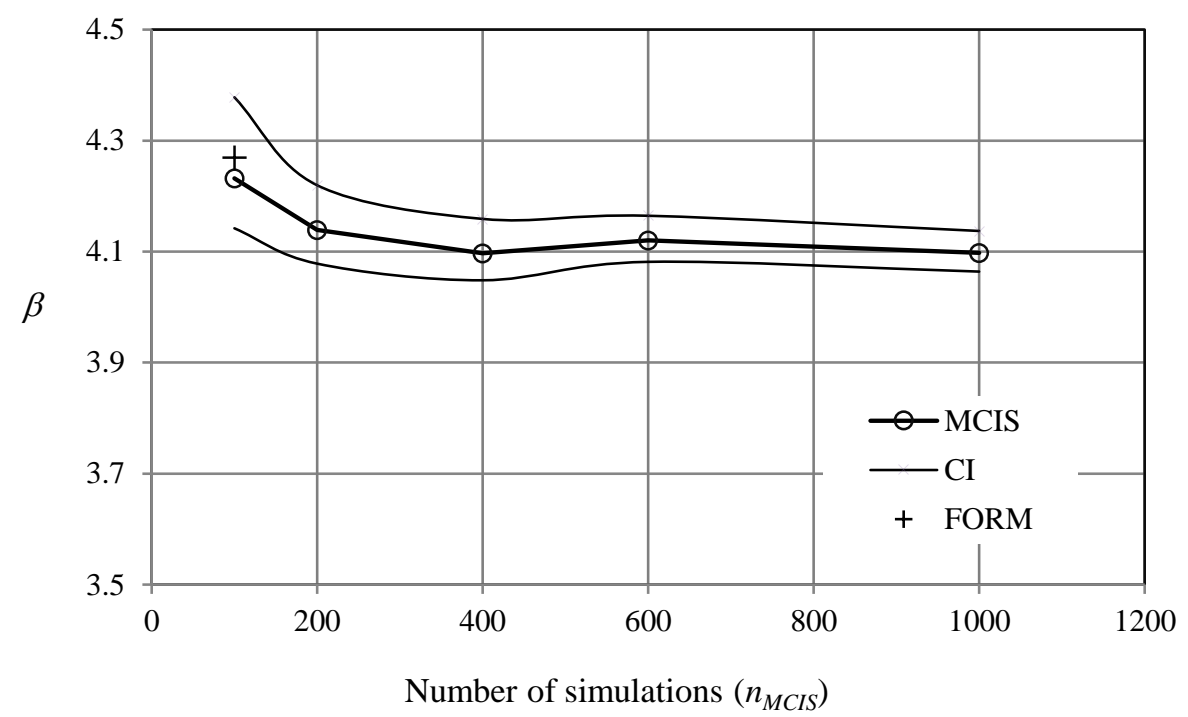

Figure 12-Results of the Monte Carlo simulation with importance sampling (MCIS) using FEA-based LSF

\subsection{Comparison of methods}

Table 8 presents a comparison of the results obtained by the different training and reliability approaches together with the number of FEAs performed in each one. The first two methods use ANN as a LSF approximator while the third method uses a FEA-based LSF. For the ANN approaches, only the most accurate results are included in the table. All methods lead to very similar results although the first method requires a much larger number of FEAs. The second and third methodologies are both very efficient despite being based on very different approaches.

The results in Table 8 demonstrate that the ANN-based reliability approach can be as efficient as the FEA-based approach for structural reliability analysis. Despite the complexity of the problem analyzed, the network can approximate the LSF very accurately and turn the MCS method a viable tool for reliability analysis of structures with low reliability level, particularly when combined with efficient adaptive training schemes.

Table 8-Comparison of the results obtained by the different training and reliability approaches

\begin{tabular}{c|c|c|c}
\hline \multirow{2}{*}{} & \multicolumn{2}{|c|}{ ANN-based LSF } & FEA-based LSF \\
\cline { 2 - 4 } & ANN $(20000)+$ MCS & $\begin{array}{c}\text { ANN (500+500) } \\
\text { +FORM+MCS with IS }\end{array}$ & FORM+MCS with IS \\
\hline$P_{f}$ & $1.97 \times 10^{-5}$ & $2.07 \times 10^{-5}$ & $2.09 \times 10^{-5}$ \\
$\beta$ & 4.111 & 4.100 & 4.098 \\
Number of & 20000 & 1000 & 1085 \\
FEAs & & & \\
\hline
\end{tabular}

\section{CONCLUSIONS}

Although great improvements on the application of artificial neural networks in reliability analyses have been made over recent years, state of art reviews on this topic are difficult to find. Therefore, this work intended to provide a wide overview on the use of ANN-based methodologies. Different nomenclatures have been used among various research groups and one of the main focuses of present work was to synthesize the subject. A chronological list showing the introduction of significant improvements on the use of ANN in structural reliability was presented. As the research effort in this field has been focused in various directions, advances 
presented in the literature were grouped in five main topics: 1) types of ANNs; 2) ANN-based methods of failure probability computation; 3) ANN training set improvement techniques; 4) comparison of ANN-based reliability methods; and 5) reliability-based structural design and optimization using ANN.

In the second part of the present work, a case study on the reliability analysis of stiffened steel plate was carried out, with the objective of comparing the efficiency of different reliability analysis approaches. The example case is a complex structural problem involving many variables and sophisticated finite element analysis, including non-linear material and geometrical behaviour. Three methods were applied, two of which use ANNs as a limit state function surrogate. The reliability assessments were performed by crude Monte Carlo simulation and with importance sampling. The ANN-based MCS is easy to implement and has very good accuracy, but the data preparation for training purposes is quite time consuming. To overcome this disadvantage, a second approach that combined ANN-based MCIS with adaptive training was introduced. In this case, the network was trained in several steps. Firstly the network was trained over the entire domain of each variable using a limited number of samples and then re-trained with an enriched set, containing additional samples from the neighbourhood of the design point. An ANN-based FORM algorithm was applied to iteratively estimate the design point. Structural reliability was computed by ANN-based MCIS, using the final network.

A third approach consisting in MCIS with LSF calculated directly by a finite element analysis, instead of ANN, was also applied. This technique can be considered very efficient and provided reliable comparison for the ANN-based methods.

The first methodology has the main benefit of making MCS a viable tool for complex structures with low probabilities of failure but the time consumed with training set preparation can be relatively high. This disadvantage can be minimized by implementing parallel computation as the calculations involved in the analysis are highly scalable. However, global ANN approximations offer a significant advantage in the context of structural design, as the same network trained over the adequate domain can be used in the analysis of similar problems with different variable values. It also can be used in conjunction with structural optimization tools to produce reliability-based optimal designs.

The second approach has demonstrated that more advanced ANN training approaches (with adaptive schemes for increasing accuracy in regions of interest) implemented with sophisticated reliability methods can be considered as efficient as the traditional reliability methods. This methodology, combining adaptive ANN, FORM and MCIS, resulted in significant computational cost reduction without loss on accuracy on the predicted probabilities of failure. A drawback of using a very specifically trained network in the neighbourhood of the most probable failure point is a loss of global approximation characteristic of the ANN models. The accuracy and efficiency of this approach is similar to a third methodology, that combined FORM with MCIS based on FEA results, used in this work as a benchmark.

The results obtained show that ANN-based reliability methodologies are robust and efficient alternatives to traditional reliability methods for the analysis of complex structures, particularly, when using advanced training set selection techniques.

\section{ACKNHOWLEDGMENTS}

This work has been funded by the Portuguese Foundation for Science and Technology (Fundação para a Ciência e a Tecnologia - FCT) under project "Adaptive methods for reliability analysis of complex structures", contract PTDC/ECM/115932/2009. 


\section{REFERENCES}

[1] Hurtado J. E., and Alvarez D. A., 2001, "Neural-network-based reliability analysis : a comparative study," Computer methods in applied mechanics and engineering, 191, pp. 113-132.

[2] Hasofer A. M., and Lind N. C., 1974, "An Exact and Invariant First-Order Reliability Format," Journal of Engineering Mechanics Div. (ASCE), 100, pp. 111-121.

[3] Rackwitz R., and Flessler B., 1978, "Structural reliability under combined random load sequences," Computers \& Structures, 9(5), pp. 486-494.

[4] Liu P.-L., and Der Kiureghian A., 1991, "Optimization algorithms for structural reliability," Structural Safety, 9(3), pp. 161-177.

[5] Fiessler B., Rackwitz R., and Neumann H., 1979, "Quadratic Limit States in Structural Reliability," Journal of the Engineering Mechanics Division, 105(4), pp. 661-676.

[6] Breitung K., 1984, "Asymptotic Approximations for Multinormal Integrals," Journal of Engineering Mechanics, 110(3), pp. 357-366.

[7] Der Kiureghian A., Lin H., and Hwang S., 1987, "Second-Order Reliability Approximations," Journal of Engineering Mechanics, 113(8), pp. 1208-1225.

[8] Tvedt L., 1990, "Distribution of Quadratic Forms in Normal Space-Application to Structural Reliability," Journal of Engineering Mechanics, 116(6), pp. 1183-1197.

[9] Bucher C. G., 1988, "Adaptive sampling — an iterative fast Monte Carlo procedure," Structural Safety, 5(2), pp. 119-126.

[10] Hohenbichler M., and Rackwitz R., 1988, "Improvement Of Second-Order Reliability Estimates by Importance Sampling,” Journal of Engineering Mechanics, 114(12), pp. 2195-2199.

[11] Shanmugam K. S., and Balaban P., 1980, "A Modified Monte-Carlo Simulation Technique for the Evaluation of Error Rate in Digital Communication System," IEEE Transactions on communications, COM-28(11), pp. 1916-1924.

[12] Bjerager P., 1988, "Probability integration by directional simulation," Journal of Engineering Mechanics, 114(8), pp. 1285-1302.

[13] Au S.-K., and Beck J. L., 2001, "Estimation of small failure probabilities in high dimensions by subset simulation," Probabilistic Engineering Mechanics, 16(4), pp. 263-277.

[14] Papadopoulos V., Giovanis D. G., Lagaros N. D., and Papadrakakis M., 2012, "Accelerated subset simulation with neural networks for reliability analysis," Computer Methods in Applied Mechanics and Engineering, 223-224, pp. 70-80.

[15] Bucher C. G., and Bourgund U., 1990, "A fast and efficient response surface approach for structural reliability problems," Structural Safety, 7(1), pp. 57-66.

[16] Rajashekhar M. R., and Ellingwood B. R., 1993, "A new look at the response surface approach for reliability analysis," Structural Safety, 12(3), pp. 205-220.

[17] Kim S.-H., and Na S.-W., 1997, "Response surface method using vector projected sampling points," Structural Safety, 19(1), pp. 3-19.

[18] Das P. K., and Zheng Y., 2000, "Cumulative formation of response surface and its use in reliability analysis," Probabilistic Engineering Mechanics, 15(4), pp. 309-315.

[19] Kmiecik M., and Guedes Soares C., 2002, "Response surface approach to the probability distribution of the strength of compressed plates," Marine Structures, 15(2), pp. 139-156.

[20] Teixeira A. P., and Guedes Soares C., 2010, "Response surface reliability analysis of steel plates with random fields of corrosion," Safety, Reliability and Risk of Structures, Infrastructures and 
Engineering Systems, Furuta, Frangopol \& Shinozuka (Eds.), Taylor \& Francis Group, London, U.K., pp. 474-481.

[21] Gaspar B., Naess A., Leira B. J., and Guedes Soares C., 2012, "System reliability analysis by Monte Carlo based method and finite element structural models," Journal of Offshore Mechanics and Arctic Engineering (in press).

[22] McCulloch W., and Pitts W., 1943, "A Logical calculus of the ideas immanent in nervous activity," Buletin of Mathematical Biophysics, 5, pp. 115-133.

[23] Hornik K., Stinchcombe M., and White H., 1989, "Multilayer feedforward networks are universal aproximators," Neural Networks, 2, pp. 359-366.

[24] Bucher C., and Most T., 2008, "A comparison of approximate response functions in structural reliability analysis," Probabilistic Engineering Mechanics, 23(2-3), pp. 154-163.

[25] Hosni Elhewy A., Mesbahi E., and Pu Y., 2006, "Reliability analysis of structures using neural network method," Probabilistic Engineering Mechanics, 21(1), pp. 44-53.

[26] Gomes H., and Awruch A., 2004, "Comparison of response surface and neural network with other methods for structural reliability analysis," Structural Safety, 26(1), pp. 49-67.

[27] Cardoso J., R. de Almeida J., Dias J., and Coelho P., 2008, "Structural reliability analysis using Monte Carlo simulation and neural networks," Advances in Engineering Software, 39(6), pp. 505513.

[28] Hurtado J. E., and Alvarez D. A., 2000, "Reliability Assessment of Structural Systems Using Neural Networks," European Congress on Computational Methods in Applied Sciences and Engineering (ECCOMAS), pp. 11-14.

[29] Bucher C., Macke M., and Most T., "Approximate response functions in structural reliability analysis," Proceedings of the fifth computational stochastic mechanics conference, Rhodos, Greece, June 21-23, 2006.

[30] Hurtado J. E., 2001, "Neural Networks in Stochastic Mechanics," Archives of Computational Methods in Engineering, 8(3), pp. 303-342.

[31] Papadrakakis M., Papadopoulos V., and Lagaros N. D., 1996, "Structural reliability analyis of elastic-plastic structures using neural networks and Monte Carlo simulation," Computer Methods in Applied Mechanics and Engineering, 136(1), pp. 145-163.

[32] Papadrakakis M., and Lagaros N. D., 2002, "Reliability-based structural optimization using neural networks and Monte Carlo simulation," Computer Methods in Applied Mechanics and Engineering, 191, pp. 3491-3507.

[33] Cho S. E., 2009, "Probabilistic stability analyses of slopes using the ANN-based response surface," Computers and Geotechnics, 36(5), pp. 787-797.

[34] Kingston G. B., Rajabalinejad M., Gouldby B. P., and Van Gelder P. H. A. J. M., 2011, "Computational intelligence methods for the efficient reliability analysis of complex flood defence structures," Structural Safety, 33(1), pp. 64-73.

[35] Goh A. T. C., and Kulhawy F. H., 2003, "Neural network approach to model the limit state surface for reliability analysis," Canadian Geotechnical Journal, 40(6), pp. 1235-1244.

[36] Holland J. H., 1962, "Outline for a logical theory of adaptive systems," Journal of the Association for Computing Machinery, 3, pp. 297-314.

[37] Cheng J., 2007, "Hybrid genetic algorithms for structural reliability analysis," Computers \& Structures, 85(19-20), pp. 1524-1533. 
[38] Cheng J., and Li Q., 2008, "Reliability analysis of structures using artificial neural network based genetic algorithms," Computer Methods in Applied Mechanics and Engineering, 197(45-48), pp. 3742-3750.

[39] Cheng J., 2010, “An artificial neural network based genetic algorithm for estimating the reliability of long span suspension bridges," Finite Elements in Analysis and Design, 46(8), pp. 658-667.

[40] Nie J., and Ellingwood B. R., 2004, “A new directional simulation method for system reliability . Part II : application of neural networks," Probabilistic Engineering Mechanics, 19(3), pp. 437-447.

[41] Anjum M. F., Tasadduq I., and Al-Sultan K., 1997, "Response surface methodology: A neural network approach," European Journal Of Operational Research, 101, pp. 65-73.

[42] Pu Y., and Mesbahi E., 2006, "Application of artificial neural networks to evaluation of ultimate strength of steel panels," Engineering Structures, 28, pp. 1190-1196.

[43] Shao S., and Murotsu Y., 1997, "Structural Reliability Analysis Using a Neural Network," The Japan Society of Mechanical Engineers International Journal, 40(3), pp. 242-246.

[44] Schueremans L., and Vangemert D., 2005, "Benefit of splines and neural networks in simulation based structural reliability analysis," Structural Safety, 27(3), pp. 246-261.

[45] Cheng J., Li Q., and Xiao R., 2008, “A new artificial neural network-based response surface method for structural reliability analysis," Probabilistic Engineering Mechanics, 23(1), pp. 51-63.

[46] Fang K-T, and Wang Y, 1996, Number-theoricetic methods in statistics, London: Champan \& Hall.

[47] Ren Y., and Bai G., 2011, "New Neural Network Response Surface Methods for Reliability Analysis," Chinese Journal of Aeronautics, 24(1), pp. 25-31.

[48] McKay M. D., Beckman R. J., and Conover W. J., 1979, "A Comparision of Three Methods for Selecting Values of Input Variables in the Analysis of Output from a Computer Code," Technometrics, 21(2), pp. 239-245.

[49] Olsson A., Sandberg G., and Dahlblom O., 2003, "On Latin hypercube sampling for structural reliability analysis," Structural Safety, 25(1), pp. 47-68.

[50] Deng J., Gu D., Li X., and Yue Z. Q., 2005, "Structural reliability analysis for implicit performance functions using artificial neural network," Structural Safety, 27(1), pp. 25-48.

[51] Deng J., 2006, "Structural reliability analysis for implicit performance function using radial basis function network," International Journal of Solids and Structures, 43(11-12), pp. 3255-3291.

[52] Lopes P. A. M., Gomes H. M., and Awruch A. M., 2010, "Reliability analysis of laminated composite structures using finite elements and neural networks," Composite Structures, 92(7), pp. 1603-1613.

[53] Deheeger F., and Lemaire M., 2006, "Reliability analysis using support vector machine classification," Reliability analysis using support vector machine classification.

[54] Tan X., Bi W., Hou X., and Wang W., 2011, "Computers and Geotechnics Reliability analysis using radial basis function networks and support vector machines," Computers and Geotechnics, 38(2), pp. 178-186.

[55] Deng J., Yue Z. Q., Tham L. G., and Zhu H. H., 2003, "Pillar design by combining finite element methods, neural networks and reliability: a case study of the Feng Huangshan copper mine, China," Rock Mechanics, 40, pp. 585-599.

[56] Zhang J., and Foschi R. O., 2004, "Performance-based design and seismic reliability analysis using designed experiments and neural networks," Probabilistic Engineering Mechanics, 19, pp. 259-267. 
[57] Chau K. W., 2007, "Reliability and performance-based design by artificial neural network," Advances in Engineering Software, 38, pp. 145-149.

[58] Tsompanakis Y., and Papadrakakis M., 2004, "Large-scale reliability-based structural optimization," Structural and Multidisciplinary Optimization, 26(6), pp. 429-440.

[59] Lagaros N. D., Charmpis D. C., and Papadrakakis M., 2005, "An adaptive neural network strategy for improving the computational performance of evolutionary structural optimization," Computer Methods in Applied Mechanics and Engineering, 194(30-33), pp. 3374-3393.

[60] IACS, 2008, “Common Structural Rules for Double Hull Oil Tankers," International Association of Classification Societes Ltd, London.

[61] Hørte T., Wang G., and White N., 2007, "Calibration of the Hull Girder Ultimate Capacity Criterion for Double Hull Tankers,” pp. 235-246.

[62] Gaspar B., Teixeira a. P., Guedes Soares C., and Wang G., 2011, "Assessment of IACS-CSR implicit safety levels for buckling strength of stiffened panels for double hull tankers," Marine Structures, 24(4), pp. 478-502.

[63] Levenberg K., 1944, "A method for the solution of certain nonlinear problems in least squares," Quart. Appl. Math., pp. 164-168.

[64] More J. J., 1978, “The Levenberg-Marquardt algorithm: Implementation and theory," Lecture Notes in Mathematics, 630, pp. 105-116.

[65] Paik J. K., Kim B. J., and Seo J. K., 2008, "Methods for ultimate limit state assessment of ships and ship-shaped offshore structures: Part II stiffened plates," Ocean Engineering, 35(2), pp. 261270.

[66] DNV, 1992, "Structural Reliability Analysis of Marine Structures," Det Norske Veritas Classification, AS, p. Classification Notes No. 30.6.

[67] Shooman M. L., 1986, Probabilistic reliability: An engineering approach, McGraw-Hill, New York.

[68] Efron B., and Tibshirani R. J., 1993, An Introduction to the Bootstrap, London: Chapman and Hall.

[69] Martinez W. L., and Martinez A. R., 2002, Computational Statistics Handbook with MATLAB, Chapman and Hall/CRC.

[70] Bucher C., 2009, Computational Analysis of Randomness in Structural Mechanics, Taylor \& Francis Group, London, UK. 